\title{
Avaliação de desempenho nas práticas orçamentárias: o que a literatura nos ensina?
}

\author{
THIAGO BRUNO DE JESUS SILVA ${ }^{1}$ \\ SANDRA ROLIM ENSSLIN ${ }^{2}$ \\ CARLOS EDUARDO FACIN LAVARDA ${ }^{2}$
}

${ }^{1}$ Universidade Federal da Grande Dourados (UFGD) / Faculdade de Administração, Ciências Contábeis e Economia, Dourados - MS, Brasil

${ }^{2}$ Universidade Federal de Santa Catarina (UFSC) / Programa de Pós-Graduação em Contabilidade, Florianópolis - SC, Brasil

\section{Resumo}

A Avaliação de Desempenho (AD) nas Práticas Orçamentárias tem sido, de forma tradicional, baseada apenas em controlar e avaliar o desempenho real e o orçado. Contudo, a AD passou a incluir indicadores coerentes com os objetivos estratégicos, atender a distintas partes interessadas no âmbito interno e no externo e a discutir diferentes propósitos. Desta forma, o objetivo deste estudo analisar as características das investigações internacionais em relação a um fragmento de literatura sobre a utilização da Avaliação de Desempenho nas Práticas Orçamentárias, ao ter como intenção a geração de conhecimento e identificação de lacunas para apontar possibilidades de futuras investigações. Empregou o Knowledge Development Process-Constructivist (ProKnow-C) para fins de orientação. Oportfólio bibliográfico foi composto de 23 artigos. Como resultados, constatou-se, nas informações qualitativas no portfólio bibliográfico, a necessidade de (i) utilizar outros paradigmas nas investigações; (ii) abordar a execução do orçamento para se obter a Avaliação de Desempenho; (iii) projetar a Avaliação de Desempenho nas Práticas Orçamentárias para atender às necessidades de amplo público; e (iv) buscar abranger mais propósitos de Avaliação de Desempenho nas Práticas Orçamentárias.

Palavras-chave: Avaliação de desempenho. Práticas orçamentárias. Lacunas. Oportunidades de pesquisa. 


\title{
Performance assessment in budgetary practices: what does the literature teach us?
}

\begin{abstract}
Performance assessment (PA) in budgetary practices has traditionally focused on monitoring and evaluating actual and budgeted performance. However, PA started to consider indicators consistent with the organization's strategic objectives to serve different internal and external stakeholders and discuss different purposes. This study aims to analyze the characteristics of the international investigations on a fragment of literature dedicated to performance assessment in budgetary practices, generating knowledge, and identifying gaps to be addressed in future research. The study adopted the Knowledge Development Process-Constructivist (ProKnow-C), and the bibliographic portfolio was composed of 23 articles. As results, the qualitative information in the bibliographic portfolio suggested the need to (i) use other paradigms in the investigations, (ii) address the budget execution to conduct performance assessment, (iii) design the performance assessment in budget practices to meet the needs of a broad public, and (iv) encompass more purposes of performance evaluation in budgetary practices.
\end{abstract}

KEYWORDs: Performance assessment. Budgetary practices. Gaps. Search Opportunities.

\section{Evaluación de desempeño en las prácticas presupuestarias: ¿qué nos enseña la literatura?}

\section{Resumen}

La evaluación de desempeño (ED) en las prácticas presupuestarias tradicionalmente se ha basado solo en controlar y evaluar el desempeño real y lo presupuestado. Sin embargo, la ED pasó a incluir indicadores coherentes con los objetivos estratégicos, a atender a distintas partes interesadas en el ámbito interno y externo y a discutir diferentes propósitos. Así, el objetivo de este estudio es analizar las características de las investigaciones internacionales en relación con un fragmento de literatura acerca de la utilización de la evaluación de desempeño en las prácticas presupuestarias, al tener como intención la generación de conocimiento e identificación de lagunas para señalar posibilidades de futuras investigaciones. Se empleó la metodología ProKnow-C (proceso de desarrollo del conocimiento constructivista) con fines orientativos. El portafolio bibliográfico estuvo compuesto por 23 artículos. Como resultado, se constató, en la información cualitativa del portafolio bibliográfico, la necesidad de (i) utilizar otros paradigmas en las investigaciones; (ii) abordar la ejecución del presupuesto para obtener la evaluación de desempeño; (iii) proyectar la evaluación de desempeño en las prácticas presupuestarias para satisfacer las necesidades del amplio público; y (iiii) tratar de abarcar más propósitos de evaluación de desempeño en las prácticas presupuestarias.

Palabras clave: Evaluación de desempeño. Prácticas presupuestarias. Lagunas. Oportunidades de investigación. 


\section{INTRODUÇÃO}

O orçamento é um dos mecanismos de coordenação e controle mais importante das organizações (ARNOLD e GILLENKIRCH, 2015). No entanto, há um debate sobre o orçamento tradicional. Enquanto os proponentes afirmam que ele deve continuar a ser utilizado como foi proposto, os opositores dizem que ele deve ser convertido em novas abordagens orçamentárias (NEELY, BOURNE e ADAMS, 2003; LIBBY e LINDSAY, 2013).

Embora essas abordagens tenham soluções diferentes, elas compartilham muitas das preocupações do orçamento tradicional (JONES, 2008). Apesar dessa discussão sobre o papel do orçamento, as evidências sugerem que o "orçamento tradicional" ainda continua vivo na indústria e continuará a ser importante no futuro, com organizações que relatam compromisso de continuar com suas práticas orçamentárias (JONES, 2008).

$\mathrm{O}$ aspecto central desses debates está relacionado ao desejo de que o processo orçamentário seja eficaz no sentido de obter a maioria dos possíveis benefícios (NEELY, BOURNE e ADAMS, 2003), quais sejam: prever o futuro; auxiliar na maximização do lucro; fornecer ao gestor um meio de comunicação; calcular as recompensas; motivar os funcionários; e avaliar e controlar o desempenho investigando suas variações (JOSHI, AL-MUDHAKI e BREMSER, 2003; AHMAD, SULAIMAN e ALWI, 2003; CRUZ, 2007).

Neely, Gregory e Platts (1995) explicam que as medidas utilizadas são inadequadas, principalmente porque incentivam o curto prazo e falta foco estratégico. Além disso, não incorporam a estratégia, baseiam-se na contabilidade tradicional desatualizada, destinam-se aos gestores e possuem métricas alimentadas com dados já passados; fazem uso de medidas já passadas, como o volume de vendas, enquanto os gestores necessitam de medidas preditivas; também, são construídas de forma não contextualizada, ou seja, estão em desconformidade com as metas organizacionais, sem considerar o retorno do investimento das partes interessadas, sem construir escalas de mensuração alinhadas às atividades organizacionais e sem aporte de uma abordagem (teoria), o que conduz à perda do benefício da sinergia (GHALAYINI e NOBLE, 1996; NEELY, 1999; CHOONG, 2014).

Nesse sentido, a Avaliação de Desempenho (AD) nas práticas orçamentárias tem sido, de forma tradicional, baseada apenas em controlar e avaliar o desempenho real e o orçado (HAKTANIR e HARRIS, 2005). Contudo, a AD passou a incluir indicadores coerentes com os objetivos estratégicos, atender a distintas partes interessadas no âmbito interno e no externo e a discutir diferentes propósitos (NEELY, GREGORY e PLATTS, 1995; BEHN, 2003; CHOONG, 2014).

A AD sofreu influências e interações com diversas áreas de gestão durante a sua evolução, e, desta forma, algumas tendências foram identificadas, como orientação estratégica dos modelos, multidimensionalidades destes de incorporar a complexidade organizacional, inclusão de aspectos externos e partes interessadas. Os modelos mais atuais são associados com o campo estratégico, no qual o desafio é facilitar a gestão das informações (CARNEIRO-DA-CUNHA, HOURNEAUX JUNIOR e CORRÊA, 2016).

Em virtude desse contexto e dada a importância da Avaliação de Desempenho às Práticas Orçamentárias, surge o seguinte problema de pesquisa: Como o conhecimento resultante da análise da literatura sobre Avaliação de Desempenho pode ser sintetizado em contribuições nas Práticas Orçamentárias? Nesse ínterim, o objetivo foi analisar as características das 
investigações internacionais em relação a um fragmento de literatura sobre a utilização da AD nas Práticas Orçamentárias, ao ter como intenção a geração de conhecimento e identificação de lacunas para apontar possibilidades de futuras investigações. Para conduzir o processo de seleção das publicações, adotou-se o Knowledge Development Process-Constructivist (Proknow-C) (DUTRA, RIPOLL-FELIU, GINER FILLOL et al., 2015; THIEL, ENSSLIN e ENSSLIN, 2017) como instrumento teórico de intervenção devido à sua abordagem científico-construtivista, alinhada ao objetivo da pesquisa e a seu processo estruturado.

Essa investigação se justifica quanto à importância, originalidade e viabilidade (CASTRO, 1977). A importância é devido à contribuição à comunidade científica e prática que estuda práticas orçamentárias com o intuito de destacar, em um único estudo, a investigação do conteúdo dos estudos ao longo dos anos, as instituições responsáveis e as principais críticas encontradas em um conjunto de obras internacionais (artigos), reconhecidas cientificamente, e relevantes ao esclarecer as bases que informam a temática e o que seus resultados representam. Considera-se original por não terem, na literatura consultada, investigações que se propusessem a apresentar as características desses estudos. Nesse sentido, percebe-se ser viável pelo acesso aos dados, ao considerar que os artigos analisados são buscados no Portal de Periódicos da CAPES e por demandar apenas o envolvimento dos pesquisadores para análise dos artigos.

\section{REFERENCIAL TEÓRICO}

\section{Avaliação de desempenho}

Para Ghalayini e Noble (1996), a AD teve duas fases principais: início de 1880 até os anos de 1980, na qual a ênfase recaía nas medidas financeiras; a segunda fase iniciou no final de 1980, na qual a mudança no comércio globalizado e competitivo demandou que as organizações refletissem sobre suas prioridades estratégicas, implementassem novas tecnologias e filosofias de gestão.

Segundo Bititci, Garengo e Nudurupati (2015), a mensuração e o gerenciamento do desempenho são um tópico que se tornou importante para a academia e para a prática, o que ficou evidente desde a revisão apresentada por Neely (2005) e até a recente revisão que foi apresentada por Choong (2014).

Nesse contexto, Neely, Gregory e Platts (1995) afirmam que a AD é um tema discutido, contudo raramente seja definido. Para Lebas (1995), medir é complexo difícil, desafiador, importante e mal utilizado. Neely, Gregory e Platts (1995) definem a AD como uma atividade complexa que consiste em um processo de quantificação da eficiência e da eficácia das ações, por meio da análise de um conjunto de indicadores individuais que interagem com o ambiente, denominados Sistemas de Avaliação de Desempenho (SADs). Vale destacar que esta investigação se afilia a essa definição teórica.

Globerson (1985) apresenta alguns critérios centrais dos indicadores de um SAD: necessita ser definido com base nos objetivos da companhia; deve permitir a comparação com outras organizações de atividades semelhantes; deve ser não ambíguo; e a organização deve ter seu controle. Para Lebas (1995), as medidas de desempenho devem, principalmente, capacitar e envolver as partes interessadas; criar base de discussão, o que proporciona melhoria contínua; 
e, sobretudo, apoiar a decisão. No âmbito da Contabilidade Gerencial, um SAD é considerado sinônimo de planejamento e orçamento (OTLEY, 1999). Contudo, Cosenz e Noto (2016) sugerem que os indicadores do SAD devem ser em número adequado, de forma que avaliem o desempenho dos processos de tomada de decisão da organização.

Segundo Bourne, Mills, Wilcox et al. (2000), o desenvolvimento do SAD deve ser concebido nestas três fases: desenho das medidas de desempenho (derivadas da estratégia organizacional); execução das medidas; e utilização das medidas de desempenho. A essas fases acrescenta-se a revisão de todas elas. Após o desenvolvimento do $\mathrm{SAD}$, este deve ser implementado, que vai interagir com o ambiente interno e o externo. No entanto, Bourne, Mills, Wilcox et al. (2000) explicam que fatores específicos, como o comprometimento do gerente e o envolvimento de especialistas em TI, são necessários para alinhar continuadamente o SAD com a estratégia. Quando combinados, esse Sistema pode atingir melhor nível.

Maskell (1991) declara que o SAD deve incorporar medidas não financeiras que necessitam ser adotadas, oferecer feedback rápido e ser projetado de modo a estimular o contínuo processo de melhoria em vez de apenas monitorar.

Com base nas manifestações desses teóricos e nos citados na sequência, conclui-se que a AD tradicional tem muitas limitações. Segundo (i) Neely, Gregory e Platts (1995), as medidas utilizadas são inadequadas, sobretudo por que elas incentivam o curto prazo e falta foco estratégico; (ii) conforme Ghalayini e Noble (1996), a AD não incorpora a estratégia, tem base na contabilidade tradicional desatualizada, é destinada aos gestores e possui métricas alimentadas com dados já passados; (iii) de acordo com Neely (1999), ela faz uso de medidas já passadas, como o volume de vendas, enquanto os gestores necessitam de medidas preditivas; (iv) segundo Choong (2014), a AD é construída de forma não contextualizada, ou seja, está em desconformidade com as metas organizacionais, sem considerar a relevância das partes interessadas, sem construir escalas de mensuração alinhadas às atividades organizacionais e sem aporte de uma abordagem (teoria) que conduz à perda do benefício da sinergia. Nesse contexto, os autores mencionados concluem que os gestores utilizam a AD para monitorar e procurar medidas preditivas que mostram que algo está fora do controle. Entretanto, Behn (2003) afirma que a $\mathrm{AD}$ pode ser útil para avaliar, controlar, orçar, motivar, promover, celebrar e aprender o que melhorar.

Assim, manter o alinhamento do SAD com a estratégia organizacional não é tarefa simples. Leva tempo, recurso e energia para reformular as métricas, o que também considera tempo para que essas mudanças sejam comunicadas, assimiladas e postas em prática na organização (MELNYK, BITITCI, PLATTS et al., 2014). Considerando-se que os comportamentos (organizacionais e individuais) são moldados por sentimentos, valores, crenças e percepções dos indivíduos, da organização e dos stakeholders, conforme Bititci, Garengo, Dörfler et al. (2012), toda e qualquer reformulação e mudança impacta e é impactada pelo comportamento dos indivíduos. De onde depreende-se que a $\mathrm{AD}$ é um fenômeno social.

Bititci, Garengo, Dörfler et al. (2012) explicam que esse campo se desenvolveu ao longo de diversas fases, como controle orçamentário, gerenciamento de produtividade, avaliação integrada e gerenciamento de desempenho integrado. $O$ conhecimento na área sofreu influências e interações com diversas áreas de gestão durante a sua evolução, e, dadas a isso, algumas tendências foram identificadas, como orientação estratégica dos modelos, multidimensionalidades destes de incorporar a complexidade organizacional, inclusão de 
aspectos externos e partes interessadas. Os modelos atuais estão associados com a estratégia, na qual o desafio é facilitar a gestão das informações que esses modelos geram (CARNEIRO-DACUNHA, HOURNEAUX e CORRÊA, 2016).

Nesse sentido, há três conclusões: a quantidade de pesquisas feitas mostra o interesse geral no assunto; a quantidade de perguntas deixadas sem resposta dá uma indicação da complexidade da medição de desempenho como um tópico de pesquisa; ou aponta para um número de problemas mais fundamentais que inibem um progresso efetivo do campo (VAN CAMP e BRAET, 2016). Bititci, Garengo, Dörfler et al. (2012) concluíram que a comunidade de pesquisa é ciente dos desafios, contudo tem dado pouca atenção a essa complexidade. Essas tendências irão mudar a forma como se mede, gerencia e avalia o desempenho. A realização de pesquisas empíricas também representa outro desafio.

\section{Avaliação de desempenho nas práticas orçamentárias}

Segundo Arnold e Gillenkirch (2015), os orçamentos, costumam ser utilizados simultaneamente para objetivos distintos de planejamento e avaliação de desempenho. As suas utilidades também podem ser descritas como (1) planejamento (planejamento, coordenação, alocação de recursos e determinação dos volumes operacionais) e (2) diálogo (comunicação, criação de consciência e motivação), como discutido por Silva e Lavarda (2020). Para Bruns e Waterhouse (1975), o orçamento pode ser definido como planos financeiros que fornecem base para direcionar e avaliar o desempenho dos indivíduos ou das organizações.

Horngren, Datar e Foster (2006) definem o orçamento como a expressão quantitativa de um plano de ação proposto pela administração por um período específico e uma ajuda para coordenar o que precisa ser feito para implementar esse plano. Blumentritt (2006, p. 73) coloca o orçamento como "o processo de alocar os recursos financeiros de uma organização às suas unidades, atividades e investimentos”. Na literatura, o orçamento participativo é chamado de abordagem "de baixo para cima"; e o orçamento autoritário é chamado de abordagem "de cima para baixo" (JONES, 1998, 2008).

Durante o processo orçamentário, se as metas dadas aos colaborares são muito difíceis de cumprir, podem levar a algumas ações orçamentárias de jogo, como estimativas mais baixas de vendas para tornar a meta de orçamento mais fácil de atingir e gastar todo o orçamento no fim do período orçamentário para não perder seu "direito" quando o próximo orçamento for estabelecido, e assim por diante (LIBBY e LINDSAY, 2013). Para evitar essas ações de jogo, as metas devem ser racionais e definidas pela comunicação com os subordinados. Em outras palavras, a participação no processo orçamentário é fundamental para evitar ações de jogo. Outros benefícios da participação impede a assimetria de informações entre altos gerentes e gerentes de nível inferior, proporciona motivação aos chefes de departamento e comitê, o que aprimora as atitudes de trabalho dos funcionários e resulta em nível maior de comprometimento de metas por gerentes de nível inferior (YUEN, 2007).

Contudo, existe a adoção de outras práticas para medir, controlar, gerenciar e avaliar o desempenho, tais como: previsões contínuas, Balanced Scorecard, custeio baseado em atividades, gerenciamento baseado em atividades (CRUZ, 2007). Otley (1999) explica que, em contabilidade gerencial, um SAD é considerado sinônimo de planejamento e orçamento. 
Nesse ínterim, Arnold e Gillenkirch (2015) explicam que os estudos contribuíram para a compreensão dos efeitos do orçamento utilizados para a AD. Entretanto, Franco-Santos, Kennerley, Micheli et al. (2007) argumentam que o campo da AD deve se desenvolver, tornar-se mais relevante entre a teoria e a prática, e os pesquisadores necessitam ser mais específicos e explícitos sobre as características dos sistemas, pois compromete a generalização e comparabilidade das pesquisas.

Para Neely, Bourne e Adams (2003), o tema predominante na literatura é que os processos de planejamento e orçamento tradicionalmente usados em muitas organizações não conseguem entregar resultados. O problema é que eles agregam valor limitado ao gerenciamento de negócios. Eles são muito demorados e dispendiosos para empreender e incentivam a política interna e o comportamento de jogo, em vez de impulsionar o desempenho dos negócios. Além disso, um dos maiores problemas com orçamentos é que eles tendem a promover uma cultura de curto prazo voltada para dentro, que se concentra em atingir um orçamento, em vez de implementar a estratégia de negócios e criar valor para o acionista a médio e longo prazos. Grande parte da literatura argumenta que, coletivamente, essas fraquezas levam ao mau desempenho dos negócios.

Para serem eficazes, os orçamentos devem estar alinhados com as estratégias da organização, com os processos apropriados de planejamento estratégico e gestão de desempenho introduzidos. Devem, também, envolver processos baseados em valores, ou seja, focados em identificar e gerenciar os impulsionadores do valor do acionista, que explicitam as ligações entre esses impulsionadores de valor e que promovam um processo contínuo de questionamento e contestação das premissas inerentes à estratégia. Ainda assim, poucos atendem a esses critérios de eficácia (NEELY, BOURNE e ADAMS, 2003).

\section{PROCEDIMENTOS METODOLÓGICOS}

\section{Enquadramento metodológico, procedimentos para coleta de dados e instrumento de intervenção}

A presente investigação, de acordo com o problema, se caracteriza como qualitativa; e, quanto ao objetivo, se caracteriza como descritiva e exploratória. Para coleta de dados, foram utilizados dados primários e secundários. Assim, a seleção de artigos, para formação do Portfólio Bibliográfico $(\mathrm{PB})$, usa dados primários, visto que as delimitações são feitas pelos investigadores em todas as circunstâncias necessárias durante o processo e na análise realizada. A análise bibliométrica, nesse sentido, utiliza dados secundários ao identificar e analisar os destaques das características que são extraídas do PB selecionado.

Vale destacar que se empregou o ProKnow-C para fins de orientação. Nesse ínterim, o ProKnow-C objetiva promover conhecimento por meio das seguintes etapas: (i) seleção do portfólio bibliográfico (PB); (ii) análise bibliométrica; (iii) análise sistêmica; e (iv) formulação de perguntas de pesquisas (DUTRA, RIPOLL-FELIU, GINER FILLOL et al., 2015). A primeira etapa é a seleção do portfólio bibliográfico. Os resultados da operacionalização dessa etapa estão demonstrados na Figura 1. 
FIGURA 1

Etapas para Seleção do Portfólio Bibliográfico orientadas pelo ProKnow-C

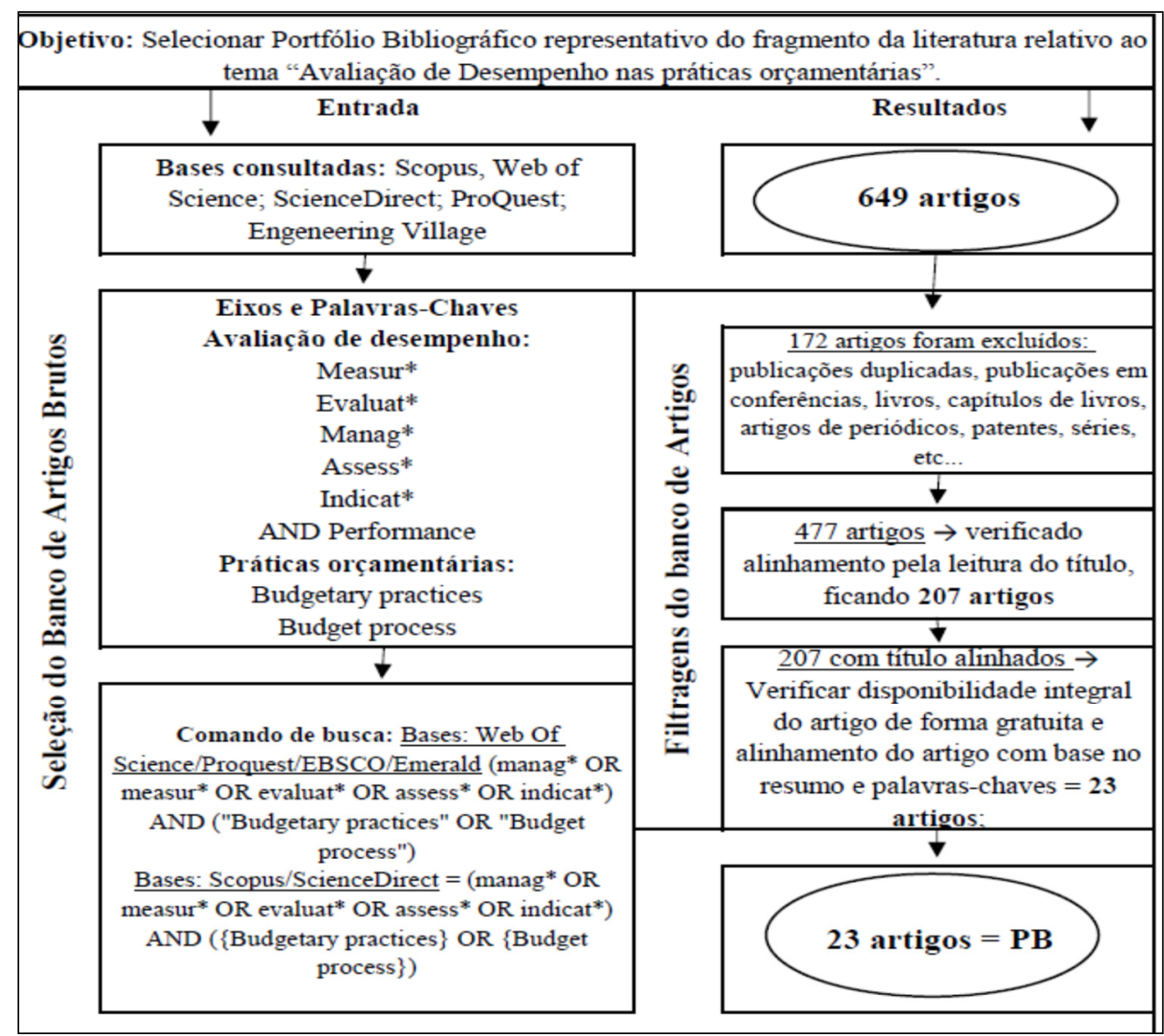

Fonte: Elaborada pelos autores.

O estudo consultou as principais bases para seleção dos artigos. A coleta dos dados ocorreu entre os dias 21 a 28 de março de 2018, e o PB final sobre o tema Avaliação de Desempenho nas Práticas Orçamentárias selecionado foi de 23 artigos. Esses artigos estão listados na seção das Referências entre [ ] sinalizados com a letra E para os artigos empíricos, e com a letra T para os artigos teóricos.

\section{PROCEDIMENTOS PARA ANÁLISE DOS DADOS}

Após definido o Portfólio Bibliográfico, foi realizada a segunda etapa do ProKnow-C, análise bibliométrica, que aproxima o pesquisador do tema e lhe gera conhecimento suficiente para identificar lacunas e propor desafios para os futuros trabalhos.

A análise bibliométrica objetiva gerar conhecimento sobre pontos relevantes do tema em questão e, para tanto, são selecionadas algumas características do PB, o que as transforma em variáveis e detalha sua ocorrência para auxiliar as argumentações do pesquisador (THIEL, ENSSLIN e ENSSLIN, 2017). Na bibliometria básica, as variáveis investigadas no presente estudo foram: (i) evolução dos estudos ao longo dos anos; (ii) rede de colaboração entre coautores e instituições; (iii) contexto público ou privado dos estudos; e (iv) ferramentas utilizadas. 
$\mathrm{Na}$ bibliometria avançada, as variáveis investigadas foram: (i) paradigmas que nortearam os estudos: considerara-se o emprego do paradigma Positivista para analisar se o fenômeno é único, se as afirmações e generalizações independem do tempo e implicam conceitos científicos e precisos, fixos e invariantes e se existem relações de causa e efeito; e considera-se o emprego do paradigma Interpretativista para entender o mundo do ponto de vista daqueles que o vivenciam (LUKKA, 2010); (ii) elementos presentes nos conceitos utilizados pelos autores dos artigos do PB: adotou-se como base a proposta de Neely, Gregory e Platts (1995), na qual foram observadas as características e funções; (iii) os objetivos estratégicos, as relações de ambiente interno e externo e o níveis de análise realizada, segundo Neely, Gregory e Platts (1995), Behn (2003) e Choong (2014); e (iv) propósitos da Avaliação de Desempenho nas Práticas Orçamentárias, segundo Behn (2003). Por fim, evidenciaram-se oportunidades e lacunas de investigação para a comunidade acadêmica e a corporativa.

\section{ANÁLISE E DISCUSSÕES DE DADOS}

\section{Análise bibliométrica das variáveis básicas}

A primeira investigação nos artigos do $\mathrm{PB}$ diz respeito à evolução do conteúdo dos estudos ao longo dos anos.

FIGURA 2

Evolução dos estudos de Avaliação de Desempenho nas Práticas Orçamentárias

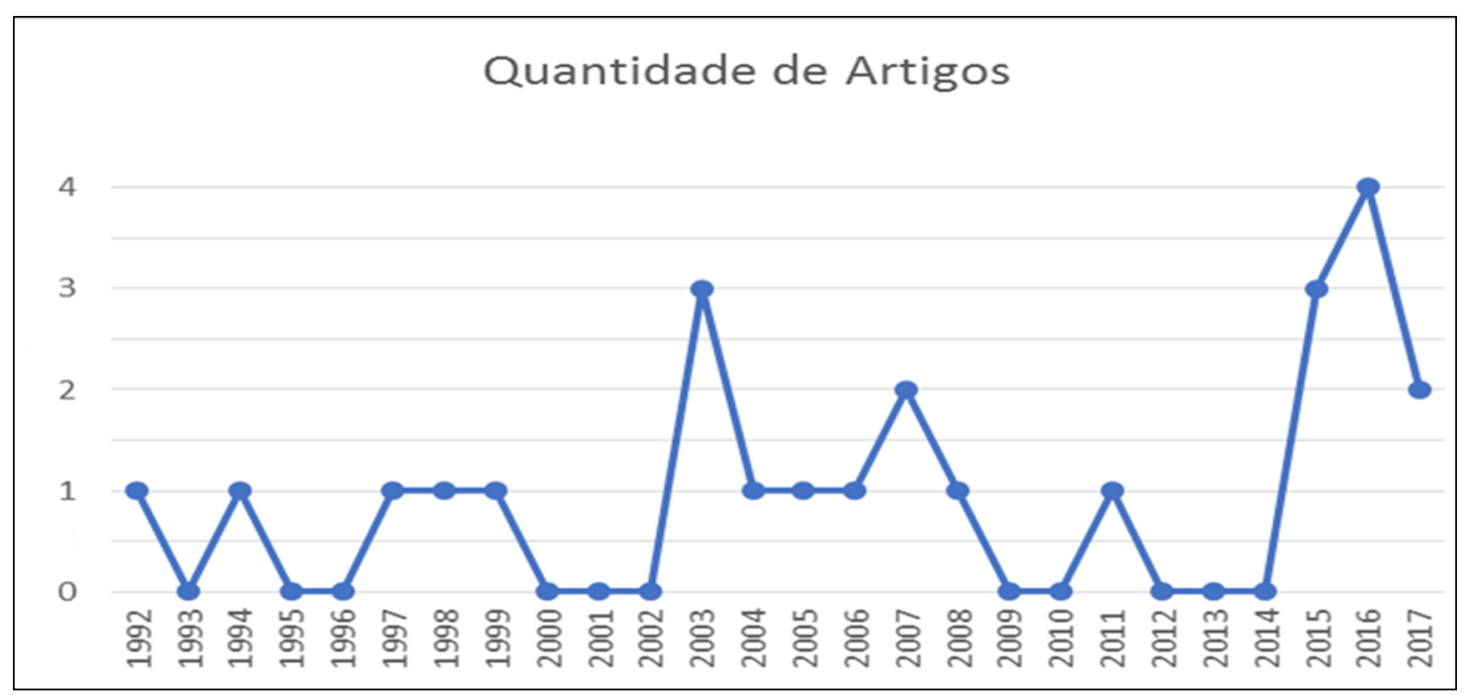

Fonte: Elaborada pelos autores.

Em linhas gerais, a evolução temporal remete à periodicidade em que os pesquisadores direcionaram esforços para identificar temas desenvolvidos e emergentes, e ainda traçar perspectiva para investigações futuras. $\mathrm{Na}$ análise temporal, Lyne (1992) foi o primeiro a pesquisar sobre a temática, examinou as percepções de diferentes grupos de usuários (contadores e gerentes) sobre o papel do orçamento, pressão orçamentária e participação orçamentária de 
acordo ao comportamento disfuncional. Percebe-se que cresceu o interesse dos pesquisadores sobre a AD nas práticas orçamentárias, tendo em vista que, entre 2014 e 2016, nove artigos foram encontrados com esse propósito, como os estudos de Kasdin (2017) que propôs um quadro analítico para avaliar orçamento e suas áreas ineficientes; o de Elhamma (2015) que analisou o impacto do tamanho da empresa na avaliação orçamentária e em desempenho; o de Leach-Lópes, Stammerjohan, Lee et al. (2015) que analisaram o efeito do conflito na participação do orçamento sobre o desempenho no trabalho em um ambiente coreano; e o de Arnold e Gillenkirch (2015) que investigaram como uma tarefa de planejamento que está em conflito com a tarefa de $\mathrm{AD}$ afeta o comportamento nas negociações orçamentárias e seus resultados.

Vale destacar que esses estudos não deram sequência a investigações anteriores e não propuseram definições. Apenas o estudo de Kasdin (2017) propôs um framework na área pública. Esses artigos se destacam por utilizar arcabouço teórico de outras áreas, como Psicologia, Sociologia e Economia na AD nas Práticas Orçamentárias. Assim, algumas agendas de INVESTIGAÇÕES para evolução do tema foram identificadas, tais como o papel do orçamento em arranjos interorganizacionais (ANESSI-PESSINA, BARBERA, SICILIA et al., 2016; DAMAYANTHI e GOONERATNE, 2017), a integração do orçamento na gestão do desempenho (ANESSI-PESSINA, BARBERA, SICILIA et al., 2016) e variáveis contingenciais como incerteza ambiental, cultura, além da estratégia e o estilo de liderança devem ser estudadas para explicar a AD no âmbito do orçamento (ELHAMMA, 2015).

Segundo Anessi-Pessina, Barbera, Sicilia et al. (2016), novas questões foram levantadas para colocar a temática de volta ao centro das atenções, que é uma área que tem sido notavelmente pouca investigada e pouca teorizada.

Na sequência, foi analisada a evolução histórica do PB. Assim, examinou-se alguma relação entre o ano de publicação e os objetivos dos artigos. Percebeu-se que os primeiros trabalhos empíricos (LYNE, 1992; JONES, 1998), na década de 1990, buscavam examinar as percepções e atitudes dos usuários sobre o orçamento e a AD. Já os trabalhos teóricos (FAUCETT e KLEINER, 1994; BARSKY e BREMSER, 1999) abordavam novos desenvolvimentos nas medidas de desempenho, como medidas não financeiras, Balanced Scorecard e Alavancas de Controle de Simons (1995).

A partir de 2000, o único estudo teórico (GREILING, 2006) abordava como a AD poderia ser um direcionador de eficiência. Neely, Bourne e Adams (2003) investigaram se as empresas adotavam as melhores práticas orçamentárias, encontrando que algumas organizações dispensaram o orçamento; e Uddin e Tsamenyi (2005) e Waal, Hermkens-Janssen e Ven (2011) investigaram a aceitação de mudanças no processo orçamentário no monitoramento do desempenho.

A partir de 2010, Kasdin, (2016) propôs um quadro analítico para avaliar o orçamento do setor público; Anessi-Pessina, Barbera, Sicilia et al. (2016) apresentaram revisão da literatura sobre orçamento público; e Damayanthi e Gooneratne (2017) revisaram a literatura sobre o controle gerencial que se baseia na perspectiva lógica como a lente teórica para entender a base atual dessa perspectiva em pesquisa de controle gerencial. Nos estudos empíricos, Elhamma (2015) examinou o tamanho da organização na AD do orçamento; Leach-López, Stammerjohan, Lee et al. (2015) e Arnold e Gillenkirch (2015) pesquisaram o conflito na participação orçamentária sobre o desempenho no trabalho; e Henttu-Aho (2016) pesquisou características facilitadoras de novas práticas e suas implicações. 
Nesse sentido, os trabalhos consideram o design, o planejamento, em detrimento da execução e do gerenciamento do orçamento para se obter a AD. Destaca-se o descompasso entre os estudos teóricos que abordam novos desenvolvimentos para a área e os estudos empíricos que apresentam discussões sobre uso, aceitação de mudanças e conflitos na participação orçamentária sobre o desempenho do trabalho.

Em seguida, analisou-se a rede de colaboração entre coautores no PB.

FIGURA 3

Rede de colaboração de autores

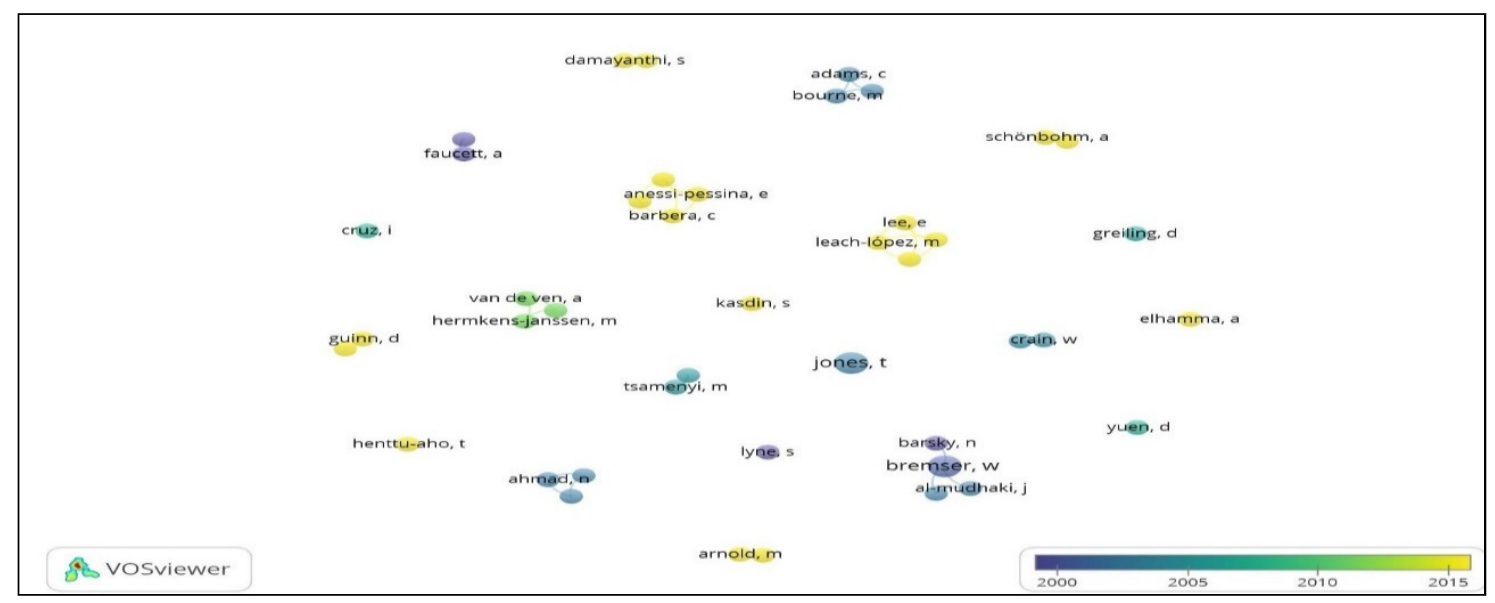

Fonte: Elaborada pelos autores.

Com relação à rede de coautores, identificaram-se 43 autores que pesquisam/pesquisaram sobre o tema. Tracy Jones e Wayne Bremser aparecem como os autores com destaque no PB, cada um com dois artigos. Tracy Jones, atualmente, é líder do grupo de Contabilidade Gerencial e de Métodos de Pesquisa e professora no curso de Contabilidade e Finanças, na University of Gloucestershire (Inglaterra). Iniciou sua carreira na área hospitalar, entretanto foi atraída pela Contabilidade e pela Avaliação de Desempenho. Possui PhD em Orçamento pela mesma universidade. Suas áreas de pesquisa são gestão e avaliação de desempenho, orçamento, hotelaria e hospitalar. Em 1998, a autora investigou o uso do orçamento nos hotéis, no Reino Unido, pois é utilizado, sobretudo, para avaliar o desempenho. Com o intuito de observar mudanças ao longo do tempo, em 2008 a autora repetiu a investigação e obteve conclusão semelhante. Vale mencionar que ela tem trabalhos, na área hospitalar, mais citados, o primeiro artigo mais citado foi publicado em 2015.

O professor Wayne Bremser, da Villanova University, teve sua formação na Drexel University, e seu Ph.D. na University of Pennsylvania. É professor de Contabilidade e Sistema de Informação. Suas áreas de pesquisa são balanced scorecard, sistema de avaliação de desempenho e inovação. O autor possui dois artigos no PB. O primeiro, datado de 1999, aborda as implicações para o orçamento e a medição do desempenho da ênfase na gestão estratégica de Recursos Humanos e de informação para obter vantagem competitiva em empresas multinacionais. No segundo artigo, em 2003, os professores PL Joshi e Jawahar Al-Mudhaki, ambos da University of Bahrain, examinaram o planejamento orçamentário, sua implementação e as práticas de Avaliação de Desempenho em organizações localizadas em Bahrain. Como artigo mais citado 
do autor, está o que aborda a utilização do balanced scorecard para medição do desempenho em P\&B, publicado em 2004. Concluída a pesquisa, constatou-se que Tracy Jones e Wayne Bremser não deram continuidade às suas investigações na área de Avaliação de Desempenho nas Práticas Orçamentárias; o que sinaliza a necessidade de novos pesquisadores na área, o que pode suscitar as ponderações de Anessi-Pessina, Barbera, Sicilia et al. (2016) quanto a novos questionamentos com a intenção de repor a temática em evidência novamente.

Com base na Figura 4, analisou-se a rede de colaboração entre as instituições. Com relação às instituições de destaque, identificaram-se 35 instituições, entre universidades e institutos de pesquisa. A universidade com mais autores e artigos é a University of Gloucestershire, do qual faz parte Tracy Jones; Universitá Cattolica Del Sacro, Bocconi School of Management, Lousiana Tech University e Ulsan National Institute of SC.

\section{FIGURA 4}

Rede de colaboração de rede entre as instituições (dos autores)

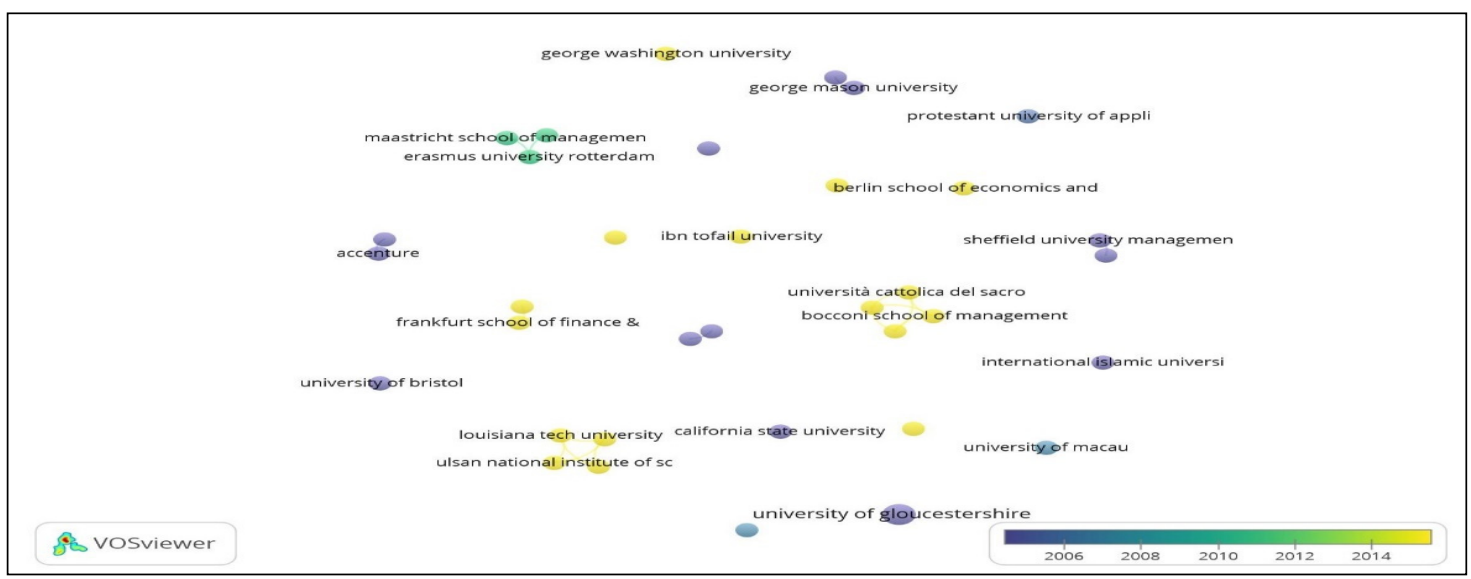

Fonte: Elaborada pelos autores.

As características do contexto organizacional, como o setor público e o setor privado, reforçam as diversas formas de abordagem da $\mathrm{AD}$ nas Práticas Orçamentárias, visto existirem aspectos que são relevantes e que ressaltam o contexto organizacional como variável que deve ser discutida.

\section{TABELA 1}

Contexto organizacional - setor público e setor privado no pb

\section{Contexto organizacional}

Setor Privado

Setor Público

\section{PB Teórico}

2

4

6

\section{PB Empírico}

13

4

17

Fonte: Elaborada pelos autores. 
No PB identificaram-se quatro estudos teóricos no setor público, dentre os oito do $\mathrm{PB}$, enquanto dois foram realizados no âmbito privado. Para Greiling (2006), acompanhar o orçamento e a AD representa mais transparência dos resultados no setor público. Esses aspectos admitem a adoção de práticas gerenciais, o que pode ter sido impulsionado pelo processo de convergência da Contabilidade Pública às Normas Internacionais. No setor privado, Damayanthi e Gooneratne (2017) explicam que fatores sociais (como a teoria institucional) e de campo impactaram nas práticas de controle de gestão, o que influencia os SADs nas Práticas Orçamentárias, o que aumentaram o interesse de pesquisadores. Ao considerar os 17 artigos empíricos do $\mathrm{PB}, 13$ foram realizados em empresas privadas e quatro no setor público. Assim, foram realizados, no contexto privado, 15 estudos, e oito, no setor público. Esses estudos se diferem quanto à abordagem. Os artigos do setor público buscam identificar a mudança no controle orçamentário, o monitoramento do desempenho, bem como políticas e percepções errôneas no orçamento. No setor privado, os estudos buscam identificar o uso do orçamento e a relação de conflito na participação do orçamento e do desempenho.

Quanto às ferramentas utilizadas por esses estudos do PB, 05 artigos não apresentaram ou fizeram menção a alguma ferramenta, instrumento ou indicadores. No entanto, 18 estudos apresentaram. Como ferramentas mencionadas entre os estudos teóricos, tem-se o Valor Econômico Agregado, Balanced Scorecard e Alavancas de Simons (BARSKY e BREMSER, 1999) e Quadro Analítico para Avaliar o Orçamento Público (KASDIN, 2017). No âmbito empírico, há as investigações que utilizavam a Variável de Desempenho Orçamentário (LEACH-LÓPEZ, STAMMERJOHAN, LEE et al., 2015), Orçamento Baseado em Atividades (NEELY, BOURNE e ADAMS, 2003), Modelo de Medidas de Avaliação Contábil (ELHAMMA, 2015), Gerenciamento Baseado em Valor (NEELY, BOURNE e ADAMS, 2003), Balanced Scorecard (JONES, 1998, 2008; CRUZ, 2007) e Estrutura de Adoção Evolutiva (WAAL, HERMKENS-JANSSEN e VEN, 2011).

\section{Análise bibliométrica das variáveis avançadas}

Primeiro, buscou-se identificar, nos artigos teóricos e nos empíricos do $\mathrm{PB}$, os paradigmas de investigação (Positivista, Interpretativista e Crítico).

Foram analisados os artigos teóricos do PB (23), ao ser identificada a predominância do paradigma Positivista (17); o paradigma Interpretativista é identificado apenas em seis investigações.

TABELA 2

Paradigma de pesquisa - setor público e setor privado no pb

\begin{tabular}{lcccc} 
& \multicolumn{2}{c}{ PB Teórico } & \multicolumn{2}{c}{ PB Empírico } \\
\hline Paradigma de pesquisa & Privado & Público & Privado & Público \\
\hline Positivista & 1 & 3 & 11 & 2 \\
\hline Interpretativista & 1 & 1 & 2 & 2 \\
Crítico & & & & 17 \\
\hline Total & 6 & & \\
\hline
\end{tabular}

Fonte: Elaborada pelos autores. 
$\mathrm{Na}$ análise quanto aos paradigmas de pesquisa, os autores abordam, em suas investigações, a Avaliação de Desempenho nas Práticas Orçamentárias sob a perspectiva Determinista. Ou seja, consideram-se contextos organizacionais generalistas, voltados a conclusões práticas que apresentam discussões pautadas em comparação da teoria e prática. Contudo, destaca-se a necessidade de explicar os elementos analisados e antecipar situações futuras em contexto organizacional, o que apresenta as relações de causa e efeito dos objetos de estudo. Os dados demonstram, também, que os estudos na área evoluíram de forma irrisória. Assim, identifica-se o paradigma Positivista, enquanto os aspectos Interpretativistas são pouco explorados, bem como a inexistência da abordagem crítica como forma de olhar a realidade social.

Quanto aos elementos característicos das Práticas Orçamentárias do $\mathrm{PB}$, verificou-se se os estudos empíricos abordavam ferramenta ou SAD. Dos 23 artigos pertencentes ao PB, nove possuíam medidas, instrumento ou indicadores. Destes, a maior parte não apresentou um sistema integrado, mas apenas medidas individuais de desempenho, sem sinalizar se são pertencentes a algum sistema ou se a organização adotava somente aquelas medidas individuais. Destacam-se que dois apresentaram o SAD que podiam ser examinados pelas medidas individuais e por conjunto. As ferramentas encontradas foram Participação Orçamentária Desejada (BPD, em inglês) e Balanced Scorecard.

Nos estudos, a análise dos termos comumente utilizados indica que não existe um consenso de seus significados e, por vezes, o sentido é o mesmo para coisas diferentes (CHOONG, 2014). Os artigos que apresentaram somente medidas individuais de desempenho trataram dos indicadores de lucro operacional, comparações orçada e executada e volume de vendas. Esses indicadores mensuravam o desempenho da variável ao qual estavam relacionados, o que fornecia informações gerenciais. Ao analisar a forma de mensurar o desempenho - se ele apresenta diagnóstico, se compara com outro desempenho e se oferece informações à gestão (NEELY; GREGORY; PLATTS, 1995) — realizada no artigo do PB identificou-se: seis relatavam o diagnósticos do desempenho, dois buscavam oferecer informações a gestão, e um comparava o desempenho com outro.

Estes verificados e descritos anteriormente consideravam, na sua maioria, apenas o ambiente interno. Como exceção, Yuen (2007) afirma que a variável externa (ambiente de negócios e alterações econômicas) pode influenciar o âmbito interno. Quanto às ferramentas, BPD e Balanced Scorecard, nenhuma apresentou interação com o ambiente externo, mesmo com alguns artigos que eram do setor público.

Não foi possível verificar a existência de alinhamento dos indicadores com a estratégia ou com os objetivos da empresa, critério citado como importante por Neely, Gregory e Platts (1995) e Choong (2014). A maioria dos artigos não apresentou informações suficientes sobre os objetivos estratégicos das organizações. Entre os três estudos que possuíam alinhamento, Faucett (1994) afirma que gestão de programas públicos, de igual forma o setor privado, deve medir o desempenho para atingir metas e objetivos; Yuen (2007) assevera que o desempenho no trabalho é afetado pela estratégia do negócio; e Henttu-aho (2016) apresentou a evidência que o controller do grupo tinha compreensão das prioridades estratégicas e conseguiu construir alguns cenários de metas para serem discutidos com a gerência.

Quanto ao público para o qual se comunica a AD (CHOONG, 2014), a maioria dos artigos não fornece informações suficientes para essa análise. Algumas investigações reconhecem que a $\mathrm{AD}$ deve considerar as diversas partes interessadas em sua fundamentação teórica 
(NEELY, BOURNE e ADAMS, 2003; JOSHI, AL-MUDHAKI e BREMSER, 2003; AHMAD, SULAIMAN e ALWI, 2003; CRUZ, 2007; LIBBY e LINDSAY, 2013), contudo essas pesquisas abordam o âmbito interno como colaboradores e/ou gestor (ARNOLD e GILLENKIRCH, 2015; LEACH-LÓPEZ, STAMMERJOHAN, LEE et al., 2015).

No setor público, a Avaliação de Desempenho nas Práticas Orçamentárias é voltada a governo (CRAIN e O'ROARK, 2004; GREILING, 2006; KASDIN, 2017), sociedade (FAUCETT, 1994; CRAIN e O'ROARK, 2004; GREILING, 2006), colaboradores (FAUCETT, 1994; CRAIN e O’ROARK, 2004; GREILING, 2006), gestor público (FAUCETT, 1994) e político (UDDIN e TSAMENYI, 2005). Nesse sentido, dada a diversidade de partes interessadas, deve-se projetar para atender às necessidades de amplo público (CHOONG, 2014).

Com base na Tabela 3, analisou-se os propósitos da AD, segundo Bhen (2003), nas práticas orçamentárias.

\section{TABELA 3}

\section{Propósitos da avaliação de desempenho nas práticas orçamentárias} - setor público e setor privado no PB

\begin{tabular}{|c|c|c|c|c|}
\hline \multirow[t]{2}{*}{ Propósitos } & \multicolumn{2}{|c|}{ Fonte (Teóricos - T) } & \multicolumn{2}{|r|}{ Fonte (Empíricos - E) } \\
\hline & Público & Privado & Público & Privado \\
\hline Avaliar & T1;T5; T6 & $\mathrm{T} 2$ & $\begin{array}{l}\text { E3; E6; E15; } \\
\text { E17 }\end{array}$ & $\begin{array}{c}\text { E1; E2; E4; E5; E7; E8; E9; E10; E11;E12;E13; } \\
\text { E14; E16 }\end{array}$ \\
\hline Controlar & T1;T5;T6 & $\mathrm{T} 2$ & $\begin{array}{l}\text { E3; E6; E15; } \\
\text { E17 }\end{array}$ & $\begin{array}{c}\text { E1;E2; E4;E5;E7; E8; E9; E10; E11;E12; E13; } \\
\text { E14; E16 }\end{array}$ \\
\hline Orçar & T1; T5; T6 & $\mathrm{T} 2$ & $\begin{array}{l}\text { E3; E6; E15; } \\
\text { E17 }\end{array}$ & $\begin{array}{c}\text { E1;E2;E4;E5;E7; E8; E9; E10; E11;E12; E13; } \\
\text { E14; E16 }\end{array}$ \\
\hline Motivar & & $\mathrm{T} 2$ & E17 & E2; E4; E8; E11; E12; E13 \\
\hline Promover & & $\mathrm{T} 2$ & E17 & \\
\hline \multicolumn{5}{|l|}{ Celebrar } \\
\hline Aprender & & $\mathrm{T} 2$ & E17 & \\
\hline Melhorar & T1; T5; T6 & $\mathrm{T} 2$ & E3; E6; E17 & E2; E3; E4; E8;E9;E11;E12;E13 \\
\hline
\end{tabular}

Fonte: Elaborada pelos autores.

Em relaçãoaos estudos realizados nosetor público, os propósitos da Avaliação de Desempenho nas Práticas Orçamentárias foram avaliar, controlar, orçar e melhorar. No setor privado, foram avaliar, controlar, orçar, motivar e melhorar. Com base nos propósitos identificados, o setor privado apresenta maiores propósitos que o setor público, conforme apontado por Behn (2003), em que o setor privado é mais abrangente que o público. Entretanto, nos artigos do PB, nenhum buscou celebrar, poucos promoveram e obtiveram aprendizagem na Avaliação de Desempenho 
nas Práticas Orçamentárias. Nesse sentido, o autor afirma que os gestores necessitam refletir sobre os fins gerenciais para que a AD contribua e possa implementar essas medidas. Dessa forma, o gestor pode utilizar medidas de desempenho para avaliar, controlar, orçar, motivar, promover, celebrar, aprender e melhorar como parte de sua estratégia de gestão.

\section{Contribuições para o avanço da área}

Ao se analisar esse fragmento da literatura científica, foi possível evidenciar oportunidades e lacunas de investigação na Avaliação de Desempenho nas Práticas Orçamentárias, para as comunidades acadêmica e corporativa, conforme demonstrado no Quadro 1. Nesse modo, esses pressupostos científicos e práticos abrem cenários para futuras pesquisas.

\section{QUADRO 1}

\section{Contribuição acadêmica e prática para pesquisas futuras}

Lacunas

provenientes

das variáveis

Básica

Avançada
Oportunidades Acadêmicas

Oportunidades Práticas

Dar sequência às investigações anteriores e propor definições.

Integrar o orçamento na gestão do desempenho.

Investigar variáveis contingenciais, cultura, estratégia e estilo de liderança para explicar a Avaliação do Desempenho nas Práticas Orçamentárias.

Abordar a execução do orçamento para se obter a AD.

Identificar o papel do orçamento em arranjos interorganizacionais.
Utilizar outros paradigmas nas
investigações.

Sinalizar a existência de SAD ou se a organização adota apenas medidas individuais

Evidenciar o ambiente para o qual a organização se reporta.

Fornecer informações para as partes com as quais a Avaliação de Desempenho nas Práticas Orçamentárias se comunica. Identificar os propósitos da Avaliação de Desempenho nas Práticas Orçamentárias.
Procurar desenvolver alianças com o ambiente acadêmico para desenvolver ou melhorar ferramentas de Avaliação de Desempenho nas Práticas Orçamentárias, conforme suas particularidades.

Fazer diagnóstico dos indicadores utilizados na Avaliação do Desempenho.

Utilizar as informações da Avaliação de Desempenho nas Práticas Orçamentárias.

Alinhar os indicadores com a estratégia ou com os objetivos da organização.

Projetar a Avaliação de Desempenho nas Práticas Orçamentárias para atender às necessidades de amplo público.

Buscar abranger mais propósitos de Avaliação de Desempenho nas Práticas Orçamentárias.

Fonte: Elaborado pelos autores. 
Destaca-se que esses horizontes de novas investigações podem levar os pesquisadores da área a formar redes de colaboração, o que minimiza a carência existente, como também enaltecer as preocupações das organizações ao visar a $\mathrm{AD}$ como instrumento para gerenciar as práticas orçamentárias.

\section{CONSIDERAÇÕES FINAIS}

Os resultados descritos quanto às variáveis básicas permitem identificar que a maioria dos estudos aborda planejamento, funções gerenciais e alocações do orçamento. Os trabalhos consideram o design em detrimento da execução e o gerenciamento do orçamento para se obter AD. Em termos de estudos teóricos e práticos, percebe-se descompasso entre eles. Os estudos teóricos abordam novos desenvolvimentos, enquanto os práticos discutem o uso do orçamento, da aceitação e das mudanças e dos conflitos na participação orçamentária sobre o desempenho no trabalho.

A análise das variáveis avançadas descreve os paradigmas adotados nas investigações, nas quais se identificou a predominância do paradigma Positivista. Nesse quesito, reforça-se a necessidade de estudos sob as lentes Interpretativista e Crítica para os avanços esperados na área, o que ressalta a pouca incidência de estudos com uso desses paradigmas. $\mathrm{O}$ estudo encontrou também que, dos 23 artigos pertencentes ao $\mathrm{PB}$, nove possuíam medidas, instrumento ou indicadores. Não foi possível verificar a existência de alinhamento dos indicadores com a estratégia ou com os objetivos da empresa. Como propósitos da Avaliação de Desempenho nas Práticas Orçamentárias, nenhum buscou celebrar, poucos promoveram e obtiveram aprendizagem.

Com o intuito de promover avanços nessa área de conhecimento, com base nas lacunas identificadas, foram elencados quais seriam os desafios para pesquisadores e gestores atenderem aos requisitos que conferem validade e legitimidade ao processo avaliativo. Entre os principais desafios, citam-se investigar variáveis contingenciais das organizações para explicar a Avaliação de Desempenho nas Práticas Orçamentárias; abordar a execução do orçamento para se obter a $\mathrm{AD}$; alinhar os indicadores com a estratégia ou com os objetivos da organização; projetar a Avaliação de Desempenho nas Práticas Orçamentárias para atender às necessidades de amplo público; e buscar abranger mais propósitos de Avaliação de Desempenho nas Práticas Orçamentárias.

Não se pretende com esta investigação esgotar a discussão sobre o assunto, ao ter em vista que outros estudos necessitam ser realizados e diferentes abordagens podem divergir dos resultados. Algumas limitações da pesquisa podem ser citadas, como a formação do PB restringir-se às publicações em periódicos indexados nas bases de dados do Portal da CAPES; a artigos escritos em língua inglesa, publicados em revistas científicas indexadas nas bases selecionadas e disponíveis gratuitamente na internet; e a análise dos artigos do $\mathrm{PB}$, com relação às variáveis investigadas, foi informada pelo julgamento e interpretação dos autores. Ao visar investigações futuras, sugere-se (i) a ampliação desta pesquisa a outras bases de dados, outros idiomas e outras características; e (ii) o desenvolvimento de trabalhos que visem preencher as lacunas, aproveitar as oportunidades e os desafios apontados. 


\section{REFERÊNCIAS}

AHMAD, N. N. N.; SUlAIMAN, M.; ALWI, N. M. Are budgets useful? A survey of Malaysian companies. Managerial Auditing Journal, v. 18, n. 9, p. 717-724, 2003.

ANESSI-PESSINA, E. et al. Public sector budgeting: a European review of accounting and public management journals. Accounting, Auditing \& Accountability Journal, v. 29, n. 3, p. 491-519, 2016. $[\mathrm{PB}][\mathrm{T} 1]$.

ARNOLD, M. M. C.; GILLENKIRCH, R. R. M. Using negotiated budgets for planning and performance evaluation: an experimental study. Accounting, Organizations and Society, v. 43, p. 1-16, 2015. [PB] [E2].

BARSKY, N. N. P.; BREMSER, W. W. G. Performance measurement, budgeting and strategic implementation in the multinational enterprise. Managerial Finance, v. 25, n. 2, p. 3-15, 1999. [PB] [T2].

BEHN, R. D. Why measure performance? Different purposes require different measures. Public administration review, v. 63, n. 5, p. 586-606, 2003.

BITITCI, U. S. et al. Performance measurement: challenges for tomorrow. International Journal of Management Reviews, v. 14, n. 3, p. 305-327, 2012.

BITITCI, U. S. et al. Value of maturity models in performance measurement. International Journal of Production Research, v. 53, n. 10, p. 3062-3085, 2015.

BLUMENTRITT, T. T.; DANIS, W. W. M. Business strategy types and innovative practices. Journal of Managerial Issues, v. 18, n. 2, p. 274-291, 2006.

BOURNE, M. et al. Designing, implementing and updating performance measurement systems. International Journal of Operations \& Production Management, v. 20, n. 7, p. 754-771, 2000.

BRUNS, W. J.; WATERHOUSE, J. J. H. Budgetary control and organization structure. Journal of Accounting Research, v. 13, n. 2, p. 177-203, 1975.

CARNEIRO-DA-CUNHA, J. J. et al. Evolution and chronology of the organizational performance measurement field. International Journal of Business Performance Management, v. 17, n. 2, p. 223$240,2016$.

CASTRO, C. M. A prática da pesquisa. São Paulo: McGraw-Hill do Brasil, 1977.

CHOONG, K. K. K. Has this large number of performance measurement publications contributed to its better understanding? A systematic review for research and applications. International Journal of Production Research, v. 52, n. 14, p. 4174-4197, 2014.

CHOONG, K. K. K. Understanding the features of performance measurement system: a literature review. Measuring Business Excellence, v. 17, n. 4, p. 102-121, 2014.

COSENZ, F.; NOTO, G. Applying system dynamics modeling to strategic management: a literature review. Systems Research and Behavioral Science, v. 33, n. 6, p. 703-741, 2016.

CRAIN, W. M.; O'ROARK, J. B. The impact of performance-based budgeting on state fiscal performance. Economics of Governance, v. 5, n. 2, p. 167-186, 2004. [PB][E3]. 
CRUZ, I. How might hospitality organizations optimize their performance measurement systems? International Journal of Contemporary Hospitality Management, v. 19, n. 7, p. 574-588, 2007. [PB] [E4].

DAMAYANTHI, S.; GOONERATNE, T. Institutional logics perspective in management control research: A review of extant literature and directions for future research. Journal of Accounting \& Organizational Change, v. 13, n. 4, p. 520-547, 2017. [PB][E3].

DUTRA, A. et al. The construction of knowledge from the scientific literature about the theme seaport performance evaluation. International Journal of Productivity and Performance Management, v. 64, n. 2, p. 243-269, 2015.

ELHAMMA, A. The relationship between budgetary evaluation, firm size and performance. Journal of Management Development, v. 34, n. 8, p. 973-986, 2015. [PB] [E5].

FAUCETT, A.; KLEINER, B. H. New developments in performance measures of public programmes. International Journal of Public Sector Management, v. 7, n. 3, p. 63-70, 1994. [PB] [T4].

FRANCO-SANTOS, M. et al. Towards a definition of a business performance measurement system. International Journal of Operations \& Production Management, v. 27, n. 8, p. 784-801, 2007.

GHALAYINI, A. M.; NOBLE, J. S. The changing basis of performance measurement. International Journal of Operations \& Production Management, v. 16, n. 8, p. 63-80, 1996.

GLOBERSON, S. Issues in developing a performance criteria system for an organization. International Journal of Production Research, v. 23, n. 4, p. 639-646, 1985.

GREILING, D. Performance measurement: a remedy for increasing the efficiency of public services? International Journal of Productivity and Performance Management, v. 55, n. 6, p. 448-465, 2006. [PB] [T5].

GUINN, D. E.; STRAUSSMAN, J. D. Improving the Budget Process in Fragile and Conflict-Ridden States: Two Modest Lessons from Afghanistan. Public Administration Review, v. 76, n. 2, p. 263-272, 2016. [PB] [E6].

HAKTANIR, M.; HARRIS, P. Performance measurement practice in an independent hotel context: A case study approach. International Journal of Contemporary Hospitality Management, v. 17, n. 1, p. 39-50, 2005.

HANSEN, S. C.; OTLEY, D. T.; VAN DER STEDE, W. A. Practice developments in budgeting: an overview and research perspective. Journal of Management Accounting Research, v. 15, n. 1, p. 95-116, 2003.

HENTTU-AHO, T.Enabling characteristics of newbudgeting practiceand the roleof controller. Qualitative Research in Accounting \& Management, v. 13, n. 1, p. 31-56, 2016. [PB] [E7].

HORNGREN, C.; DATAR, S.; FOSTER, G. Contabilitatea costurilor, o abordare managerială. Moldávia: Editura Arc, 2006.

JONES, T. A. UK hotel operators use of budgetary procedures. International Journal of Contemporary Hospitality Management, v. 10, n. 3, p. 96-100, 1998. [PB] [E8].

JONES, T. A. Changes in hotel industry budgetary practice. International Journal of Contemporary Hospitality Management, v. 20, n. 4, p. 428-444, 2008. [PB][E9]. 
JOSHI, P. L.; AL-MUDHAKI, J.; BREMSER, W. G. Corporate budget planning, control and performance evaluation in Bahrain. Managerial Auditing Journal, v. 18, n. 9, p. 737-750, 2003. [PB] [E10].

KASDIN, S. An evaluation framework for budget reforms: A guide for assessing public budget systems and selecting budget process reforms. International Journal of Public Administration, v. 40, n. 2, p. 150-163, 2017. [PB][T6].

LEACH-LÓPEZ, M. A. et al. Relationship of budget participation conflict and job performance of South Korean managers. Cross Cultural Management, v. 22, n. 4, p. 608-629, 2015. [PB][E11].

LEBAS, M. J. Performance measurement and performance management. International journal of production economics, v. 41, n. 1-3, p. 23-35, 1995.

LIBBY, T.; LINDSAY, R. M. Budgeting - an unnecessary evil. CMA Magazine, v. 77, n. 1, p. 30-30, 2013.

LUKKA, K. The roles and effects of paradigms in accounting research. Management Accounting Research, v. 21, n. 2, p. 110-115, 2010.

LYNE, S. R. Perceptions and attitudes of different user-groups to the role of the budget, budget pressure and budget participation. Accounting and Business Research, v. 22, n. 88, p. 357-369, 1992. [PB][E12].

MASKELL, B. H. Performance measurement for world class manufacturing: A model for American companies. Boca Raton: CRC Press, 1991.

MELNYK, S. A. et al. Is performance measurement and management fit for the future? Management Accounting Research, v. 25, n. 2, p. 173-186, 2014.

NAZLI NIK AHMAD, N.; SULAIMAN, M.; ALWI, N. M. Are budgets useful? A survey of Malaysian companies. Managerial Auditing Journal, v. 18, n. 9, p. 717-724, 2003. [PB] [E1].

NEELY, A. The performance measurement revolution: why now and what next? International Journal of Operations \& Production Management, v. 19, n. 2, p. 205-228, 1999.

NEELY, A. A evolução da pesquisa de medição de desempenho: desenvolvimentos na última década e uma agenda de pesquisa para a próxima. Revista Internacional de Operações e Gestão da Produção, v. 25, n. 12, p. 1264-1277, 2005. [PB] [E13].

NEELY, A.; BOURNE, M.; ADAMS, C. Better budgeting or beyond budgeting? Measuring business excellence, v. 7, n. 3, p. 22-28, 2003.

NEELY, A.; GREGORY, M.; PLATTS, K. Projeto de sistema de medição de desempenho: uma revisão de literatura e agenda de pesquisa. Jornal Internacional de Operações e Gestão de Produção, v. 15, n. 4, p. 80-116, 1995.

OTLEY, D. Performance management: a framework for management control systems research. Management Accounting Research, v. 10, n. 4, p. 363-382, 1999.

SCHÖNBOHM, A.; ZAHN, A. Reflective and cognitive perspectives on international capital budgeting. Critical Perspectives on International Business, v. 12, n. 2, p. 167-188, 2016. [PB] [E14].

SILVA, T. B. J.; LAVARDA, C. E. F. Relação da utilidade do orçamento no empoderamento e na criatividade. Contextus - Revista Contemporânea de Economia e Gestão, v. 18, n. 7, p. 81-91, 2020. 
SIMONS, R. Control in an Age of Empowerment. Harvard business review, v. 73, n. 2, p. 80-88, 1995.

THIEL, G. G.; ENSSLIN, S. R.; ENSSLIN, L. Street lighting management and performance evaluation: opportunities and challenges. Lex Localis, v. 15, n. 2, p. 303, 2017.

UDDIN, S.; TSAMENYI, M. Public sector reforms and the public interest: a case study of accounting control changes and performance monitoring in a Ghanaian state-owned enterprise. Accounting, Auditing \& Accountability Journal, v. 18, n. 5, p. 648-674, 2005. [PB] [E15].

VAN CAMP, J.; BRAET, J. Taxonomizando falhas de sistemas de medição de desempenho. Revista Internacional de Produtividade e Gestão de Desempenho, v. 65, n. 5, p. 672-693, 2016.

WAAL, A.; HERMKENS-JANSSEN, M.; VAN DE VEN, A. The evolutionary adoption framework: explaining the budgeting paradox. Journal of Accounting \& Organizational Change, v. 7, n. 4, p. 316336, 2011. [PB][E16].

YUEN, D. Antecedents of budgetary participation: enhancing employees' job performance. Managerial Auditing Journal, v. 22, n. 5, p. 533-548, 2007. [PB] [E17].

THIAGO BRUNO DE JESUS SILVA

ORCID: https://orcid.org/0000-0002-0682-0881

Doutor em Contabilidade pela Universidade Federal de Santa Catarina (UFSC); Professor do curso de Ciências

Contábeis na Universidade Federal da Grande Dourados (UFGD). E-mail: thiagobruno.silva@yahoo.com.br

\section{SANDRA ROLIM ENSSLIN}

ORCID: https://orcid.org/0000-0001-7420-8507

Doutora em Engenharia de Produção pela Universidade Federal de Santa Catarina (UFSC); Professora dos Programas de Contabilidade e Engenharia de Produção da Universidade Federal de Santa Catarina (UFSC).

E-mail: sensslin@gmail.com

\section{CARLOS EDUARDO FACIN LAVARDA}

ORCID: https://orcid.org/0000-0003-1498-7881

Doutor em Contabilidade pela Universidade de Valência; Professor do Departamento de Ciências Contábeis da Universidade Federal de Santa Catarina (UFSC) na Graduação e Pós-Graduação. E-mail: elavarda@gmail.com 